\section{$\underset{\text { hommes }}{\text { \& migrations }}$}

\section{Hommes \& migrations}

Revue française de référence sur les dynamiques

migratoires

$1313 \mid 2016$

1983 , le tournant médiatique

\title{
Les mémoires de la Marche pour l'égalité et contre le racisme
}

Dans les archives du Web

\section{Sophie Gebeil}

\section{CpenEdition}

Journals

Édition électronique

URL : http://journals.openedition.org/hommesmigrations/3574

DOI : $10.4000 /$ hommesmigrations.3574

ISSN : 2262-3353

Éditeur

Musée national de l'histoire de l'immigration

Édition imprimée

Date de publication : 1 janvier 2016

Pagination : 115-122

ISBN : 978-2-919040-34-6

ISSN : 1142-852X

Référence électronique

Sophie Gebeil, «Les mémoires de la Marche pour l'égalité et contre le racisme », Hommes \& migrations [En ligne], 1313 | 2016, mis en ligne le 01 janvier 2019, consulté le 17 mars 2020. URL : http:// journals.openedition.org/hommesmigrations/3574; DOI : https://doi.org/10.4000/ hommesmigrations.3574 


\title{
LES MÉMOIRES DE LA MARCHE POUR L'ÉGALITÉ ET CONTRE LE RACISME DANS LES ARCHIVES DU WEB
}

par SOPHIE GEBEIL, docteur en histoire contemporaine (UMR 7303 Telemme CNRS-AMU, Aix-en-Provence), ATER à l'ESPé d'Aix-Marseille université, chercheure invitée à la BnF et lauréate de la bourse Pasteur Vallery-Radot 2015-2016.

\author{
Sur Internet, la mémoire de la Marche pour l'égalité et contre \\ le racisme de 1983 est loin d'être univoque. Trente ans après \\ cet événement à la fois clé et quelque peu oublié de I'histoire \\ sociale et politique française, les acteurs de la Marche \\ continuent de porter le flambeau. Cette mémoire plurielle \\ à l'échelle nationale et locale sert de référence à nombre \\ d'engagements militants contemporains. De repoussoir \\ également pour ceux qui dénoncent la récupération politique \\ dont la Marche a fait l'objet.
}

À la fin de l'année 2012, dans le cadre d'un travail de thèse sur la fabrique numérique des mémoires de l'immigration maghrébine à partir des archives du $\mathrm{Web}^{1}$, nous avons souhaité étudier la façon dont la Marche de 1983 était donnée à voir sur la Toile. L'hypothèse était alors que la démocratisation de l'Internet, notamment liée au tournant participatif du Web au milieu des années 2000, offrait un nouvel espace d'expression mémoriel. À partir d'une recherche dans les archives du Web français de 2007 à février 2013, 25 dispositifs numériques ont été identifiés pour proposer des pistes de réflexion sur la fabrique numérique des mémoires de la Marche, en tant que construction sociale et, selon les termes de Denis Peschanski, "mise en récit publique d'un passé convoqué dans le présent et

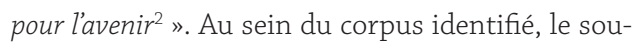
venir de l'événement oscille entre entrée en militance - et parfois en politique - d'une génération incarnée par la création de SOS Racisme en 1984 et l'amertume d'une profonde désillusion face à une Marche perçue comme confisquée par le Parti socialiste à travers l'antiracisme. Dès lors, le Web constitue un espace de coexistence de la pluralité des mémoires qui met en lumière une certaine concurrence entre les " héritiers » de la Marche ainsi que des enjeux à différentes échelles. Il s'agira ici de rendre compte d'une première approche non exhaustive des mémoires en ligne de l'événement en insistant sur quelques traits saillants des 
mécanismes de médiation du passé concernant les temporalités, les acteurs et la participation du Web au processus de mémorialisation. Le Web contribue à la présentification de l'histoire durant le temps de la commémoration et permet à une pluralité d'acteurs mémoriels de s'exprimer. Ensuite, la mémoire de l'événement s'inscrit dans les usages publics de l'histoire ${ }^{3}$ au sein de dispositifs dans lesquels le témoin occupe une place centrale. Enfin, la polyphonie mémorielle visible en ligne reflète la pluralité des parcours militants.

\section{La présentification de l'histoire sur le Web à travers l'exemple de la Marche de 1983}

La recherche des traces de la Marche de 1983 dans les archives du Web a eut lieu de décembre 2012 à février 2013. Elle s'inscrivait alors dans le cadre d'un doctorat d'histoire contemporaine visant à proposer une première approche historiographique des fonds du dépôt légal du Web géré par l'Institut national de l'audiovisuel (Ina) et la Bibliothèque nationale de France $(\mathrm{BnF})$ depuis la loi DADVSI de 2006, à partir de l'étude des mémoires de l'immigration maghrébine. Les enjeux épistémologiques liés à l'appréhension du dépôt légal du Web comme source renouvellent la relation que l'historien entretient avec ses archives et nécessitent une adaptation méthodologique sur laquelle il n'est pas ici possible de s'étendre ${ }^{4}$. Face à ces obstacles et dans le but d'analyser le Web en prenant en considération les individus qui agissent " derrière l'écran », nous avons élaboré une démarche qualitative qui s'appuyait sur un nombre restreint de treize dispositifs numériques mémoriels complétés par un faisceau de sources supplémentaires alors en cours de collecte. Une première recherche sur la Marche a été effectuée au sein de ce corpus comprenant les versions archivées des sites de l'association Génériques ${ }^{5}$, des médias de minorité du groupe Oumma Media ${ }^{6}$ et Kabyle. $\mathrm{com}^{7}$, du site muséal du Musée national de l'histoire de l'immigration ${ }^{8}$, les blogs de Touhami Moualek ${ }^{9}$ et des Arabes de France ${ }^{10}$ ainsi que les sites associatifs locaux d'Ancrages ${ }^{11}$, de Com'étik Diffusion $^{12}$ et d'AnonymalTV ${ }^{13}$. Or, en décembre 2012, la mémoire de la Marche, événement pourtant fondateur pour de nombreux acteurs, apparaissait comme secondaire, notamment au regard des commémorations de la guerre d'Algérie et du 17 octobre $1961^{14}$.

À l'approche du trentième anniversaire de la Marche, ces sites ont diffusé de nouveaux contenus sous forme de publications scientifiques ${ }^{15}$, de ressources documentaires ou pédagogiques ${ }^{16}$. Génériques $\mathrm{a}$, par exemple, mis en ligne une première

3. Marie-Claire Lavabre, "Usages du passé, usages de la mémoire ", in Revue française de science politique, vol. 44, $\mathrm{n}^{\circ}$ 3, 1994, pp. 480-493 ; Maryline Crivello, Patrick Garcia, Nicolas Offenstadt, Concurrence des passés. Usages politiques du passé dans la France contemporaine, Aix-en-Provence, Publications de l'université de Provence, 2006 ; Philippe Joutard, Histoire et mémoires, conflits et alliance, Paris, La Découverte, 2013 ; Cahiers d'histoire immédiate, $n^{\circ}$ 43, "Les usages du passé ", Toulouse, Groupe de recherche en histoire immédiate, 2013 ; Le Débat, $n^{\circ} 177$, "La culture du passé ", novembre-décembre 2013. 4. Sur l'usage des archives du Web en histoire voir notamment Valérie Schafer et Benjamin Thierry, "L'ogre et la Toile, le rendez-vous de l'histoire et des archives du Web ", in Socio, n 4, 2015 ; Sophie Gebeil, "La médiation des mémoires de l'immigration maghrébine sur le Web français de 1999 à 2014 ", in Les Cahiers du numérique, 2016. 5. Génériques, www.generiques.org, versions de 1999 à février 2013, in Archives de l'Internet, Dépôt légal du numérique, BnF. 6. Essentiellement www.oumma.com, versions de 1999 à février 2013, in Archives de l'Internet, Dépôt légal du numérique, BnF et www.oummatv.tv, versions de 2008 à février 2013, in Dépôt légal du Web média, Ina. 7. Kabyle.com, www.kabyle.com, versions de 2001 à février 2013, in Archives de l'Internet, Dépôt légal du numérique, BnF. 8. Musée d'Histoire de l'Immigration, www.histoire-immigration.fr, versions de 2004 à février 2013, in Archives de l'Internet, Dépôt légal du numérique, BnF. 9. Touhami Moualek, Blog à part, http://mtouhami.com puis http://mtouhami.fr, versions de 2006 à février 2013 , in Archives de l'Internet, Dépôt légal du numérique, BnF. 10. www.arabesdefrance.wordpress.com, versions de 2011 à février 2013 , in Archives de l'Internet, Dépôt légal du numérique, BnF. 11. Association Ancrages, www.ancrages.org, versions de 2008 à février 2013 , in Archives de l'Internet, Dépôt légal du numérique, BnF. 12. Association Com'étik Diffusion, www.cometik.info, versions de 2006 à février 2013 , in Archives de l'Internet, Dépôt légal du numérique, BnF. 13. Association Anonymal, www.anonymal.tv, versions de 2009 à février 2013, in Archives de l'Internet, Dépôt légal du numérique, BnF. 14. Commémorations marquées par l'essor de trois webdocumentaires d'envergure sur le 17 octobre 1961: Olivier Lambert et Thomas Salva, La nuit oubliée, www.lemonde.fr/societe/visuel/2011/10/17/la nuit-oubliee_1587567_3224.html ; Raspouteam, 17.10.61, http://www.raspouteam.org/1961/ ; Laurent Maffre et Thomas Gabison, 127 rue de la Garenne, http://bidonville-nanterre.arte.tv, in Dépôt légal du Web média, Ina. 15. Voir notamment les revues Hommes \& Migrations et Migrance 16. Musée d'histoire de l'immigration, http://www.histoire-immigration.fr/musee/collections/ la-marche-pour-l-egalite-et-contre-le-racisme, version du 13 mars 2014, in Archives de l'Internet, Dépôt légal du numérique, BnF. 
exposition virtuelle réalisée par l'association avec l'outil Google Open Gallery. Celle-ci concerne les luttes de l'immigration de 1972 à 1983. Présentée dans la continuité des mouvements immigrés des années 1970, la Marche de 1983 y apparaît comme le point d'orgue de la mobilisation. Ce parcours militant est présenté à travers des témoignages, dont celui de l'ancienne marcheuse MarieLaure Mahé, et des affiches dont l'association est détentrice ${ }^{17}$. Par ailleurs, comme cela avait été le cas pour les commémorations du 17 octobre 1961, deux webdocumentaires ont mis à l'honneur l'événement à l'image de La Marche d'après ${ }^{18}$ ou de 1983-2013 : une Marche, deux générations ${ }^{19}$. Dans le même temps, plusieurs pages Facebook ${ }^{20}$ et blogs ${ }^{21}$ lui sont intégralement dédiés tandis que certains acteurs développent leur identité numérique ${ }^{22}$, à l'image de Toumi Djaidja ${ }^{23}$, l'un des initiateurs de la Marche. À cela vient s'ajouter la politique communicationnelle du film La Marche, réalisé par Nabil Ben Yadir avec le soutien de Djamel Debbouze. Comme cela avait été étudié par Cyril Domanico pour Indigènes en 2006, deux axes de diffusion en ligne caractérisent la promotion du film : la réalisation d'un site $\mathrm{Web}^{24}$ et la diffusion d'un dossier pédagogique ${ }^{25}$. Dans l'ensemble, la production commémorative insiste sur l'entrée de la génération « beur » dans l'espace public et sur la personnalité des marcheurs.

\section{Une pluralité d'acteurs de la médiation de la Marche en ligne de 2002 à février 2013}

À l'inverse, les contenus identifiés en février 2013 dans les archives du Web, avant le temps de la célébration, donnent à voir des acteurs disposant de moyens financiers moindres mais relayant une mémoire très nuancée et complexe de la Marche de 1983. Le Web donne ainsi accès, aux côtés des projets commémoratifs disposant d'une forte visibilité et des associations nationales,

À l'approche du trentième à une "mémoire par le bas ", produite par des acteurs et des militants ancrés dans des territoires locaux. Le Web militant est au cœur de la mémoire anniversaire de la Marche, ces sites ont diffusé de nouveaux contenus sous forme de publications scientifiques, de ressources documentaires de 1983. Les associations et ou pédagogiques les mouvements nationaux tels que les sites de SOS Racisme ${ }^{26}$, du Mouvement de l'immigration et des banlieues (MIB ${ }^{27}$ ) mais aussi du Mouvement des Indigènes de la République $\left(\mathrm{MIR}^{28}\right)$, intègrent la Marche dans leur historique ou dans leur argumentaire, même si elle ne constitue pas un sujet central. Aux côtés de ces associations d'envergure, les mémoires de 1983 sont aussi

17. Association Génériques, https://www.google.com/culturalinstitute/exhibit/mouvements-et-luttes-des-immigré-e-s-contre-lesdiscriminations-et-pour-I-égalité/AQ-5Nl1g?hl=fr\&position=11\%2C99, disponible en ligne, consulté le 10 janvier 2015 .

18. Jenna Le Bras, François Hume-Ferkatadji, Lucas Roxo, 2013, http://www.lamarchedapres.com, in Dépôt légal du Web, INA. 19. Ouafia Kheniche, "Une marche, deux générations ", in Franceinfo.fr, 15 octobre 2013, http://www.franceinfo.fr/actu/societe/ dossier/les-30-ans-de-la-marche-27847, Dépôt légal du Web média, INA, disponible en ligne, consulté le 14 janvier 2015. 20. Voir notamment : «1983-2013, Trente ans de Marche de l'égalité », https://www.facebook.com/Marchedelegalite ; celle de Naïma Yahi, "Marche pour l'égalité et contre le racisme, 30 ans déjà ", https://www.facebook.com/Marche-pour-l\%C3\%A9galit\%C3\%A9et-contre-le-racisme-30-ans-d\%C3\%A9j\%C3\%Ao-233259286834025/timeline/; celle de la WebTV Kaina TV, https://m.facebook.com/ profile.php?id=161994420674910 ; de l'association Le comptoir général, https://www.facebook.com/events/235400929956924/, consulté le 10 septembre 2015. 21. Blog Actualités du 30 a nniversaire de la Marche pour l'égalité et contre le racisme de 1983 , Labex Passés dans le présent - ISP - BDIC - L’Echo des cités, http://marcheegalite.wordpress.com/, archivée depuis le 23 juin 2013 , Archives de l'internet, Dépôt légal du numérique, BnF, également disponible en ligne, consulté le 8 octobre 2015. 22. Fanny Georges, "Représentation de soi et identité numérique ", in Réseaux, n 154, vol. 2, 2009, pp. 165-193. 23. Toumi Djaidja, site officiel, symbole et initiateur de la marche pour l'égalité de 1983, 26 novembre 2013, https://sites.google.com/site/toumidjaidja/home, disponible en ligne, consulté le 20 octobre 2015. 24. La Marche, http://jemarche.fr/, archivé depuis le 17 novembre 2013, inactif depuis mars 2014, in Archives de l'Internet, Dépôt légal du numérique, BnF. 25. Académie de Paris, Éducation nationale, « La Marche, du film à la pédagogie ", 2 décembre 2013, https://www.ac-paris.fr/portail/jCMS/p1_844625/la-marche-du-film-a-la-pedagogie, in Archives de l'Internet, Dépôt légal du numérique, BnF, disponible en ligne, consulté le 10 septembre 2015. 26. SOS Racisme, http://www.sosracisme.org/, archivé depuis le 26 août 2000, in Archives de l'Internet, Dépôt légal du numérique, BnF. 27. MIB, http://mibmib.free.fr/, archivé depuis le 13 août 2000, in Archives de l'Internet, Dépôt légal du numérique, BnF. 28. MIR, premier site Web: http://www.indigenes-republique.org/, archivé du 19 mai 2006 jusqu'au 19 août 2011, second site Web : http://www.indigenesrepublique.fr/, archivé depuis le 17 décembre 2008, in Archives de l'Internet, Dépôt légal du numérique, BnF. 
portées par un tissu associatif local bien souvent implanté dans les quartiers populaires, émanant notamment de l'agglomération lyonnaise à travers plusieurs hommages rendus à Toumi Djaidja, habitant de la cité des Minguettes ${ }^{29}$. Le site de Radio Pluriel, radio associative et acteur historique de la Marche née en 1981, propose notamment un docu-

A partir de la sélection de vingt-trois dispositifs,

la Marche est intégrée dans un

discours plus large

et dont les horizons sont

très divers. Le Web renforce

ainsi le caractère éclaté

des mémoires dans

un espace virtuel où toutes les

informations se juxtaposent

sans hiérarchisation. mentaire radiophonique en ligne sur la Marche.

L'événement est également abordé par les médias en ligne qui entendent proposer une information alternative, en opposition aux médias traditionnel ${ }^{30}$ jugés moins libres et trop conventionnels : la WebTV généraliste Latelelibre.fr diffuse un entretien de Malek Boutih $^{31}$, les deux WebTV locales, Marsactu ${ }^{32}$ et Télé Lyon Métropole ${ }^{33}$, proposent également deux documents audiovisuels sur la Marche pour l'égalité. Deux territoires sont particulièrement représentés : Marseille, point de départ de la Marche de 1983, et Lyon, lieu de naissance de la Marche, mettant ainsi à mal une perception déterritorialisée du Web, puisque la mémoire de l'événement, relayée sur un écran ouvert au monde, émane et s'ancre malgré tout dans les territoires d'origine de la Marche. De plus, aux côtés des Web-médias généralistes et locaux, les médias de minorité en ligne ${ }^{34}$ comme Oumma. $\mathrm{com}^{35}$ et les portails destinés à la diaspora marocaine (Yabiladi.com ${ }^{36}$, Bladi.net ${ }^{37}$ ) accordent également une place non négligeable à la Marche. Le dernier type de sites Web évoquant la Marche de 1983 rassemble les plates-formes d'autopublication $^{38}$, de partage de vidéos et les réseaux socionumériques. Il existe également une page Facebook consacrée intégralement au trentième anniversaire de la Marche pour l'égalitée ${ }^{39}$. Sur YouTube, le groupe de rap ZEP s'est fait connaître en diffusant en ligne le clip de la chanson Nique la France $e^{40}$ qui cite la Marche de 1983.

À partir de la sélection de vingt-trois dispositifs, la Marche est intégrée dans un discours plus large et dont les horizons sont très divers. Le Web renforce ainsi le caractère éclaté des mémoires dans un espace virtuel où toutes les informations se juxtaposent sans hiérarchisation.

29. Rafika Bendermel, « Rencontre avec Toumi Djaidja, fondateur de la Marche pour l'égalité et contre le racisme », Lyon BondyBlog, 4 mai 2009, http://yahoo.lyon.bondyblog.fr/news/rencontre-avec-toumi-djaidja-fondateur-de-la-marche-pour-l-egalite-et-contrele-racisme, version du 17 mai 2010, in Archives de l'Internet, Dépôt légal du numérique, BnF. Voir également Olivier Minot, 25 ans de marche, et toujours pas d'égalité, http://www.crancra.org/emissions/radio-pluriel/25ansdeMarcheDocu.mp3, consulté le 10 mai 2013. 30. Colloque " 1983 " organisé par l'ANR ECRIN à Paris les 25 et 26 mars 2015, Paris. 31. John Paul Lepers, « Malek Boutih, Minorité invisible ", in LaTeleLibre.fr, $1^{\text {er }}$ septembre 2007, http://latelelibre.fr/libre-posts/malek-boutih-minorite-invisible/, version du 10 septembre 2007, in Dépôt légal du Web média, INA. 32. Esther Griffe, "Nous ne marcherons plus ", un appel pour l'égalité dans les quartiers populaires, http://www.marsactu.fr/politique/nous-ne-marcherons-plus-un-appel-pour-legalite-dansles-quartiers-populaires-26669.html, consult? le 10 mai 2013. 33. Télé Lyon Métropole, « Marche des Beurs : marche pour l'égalité ! ", Dailymotion, 8 juillet 2009, http://www.dailymotion.com/video/xgsyud_marche-des-beurs-marche-pour-I-egal_news, in Dépôt légal du Web média, INA, disponible en ligne, consulté le 10 septembre 2015. 34. Isabelle Rigoni, «Éditorial. Les médias des minorités ethniques. Représenter l'identité collective sur la scène publique ", in Revue européenne des migrations internationales, vol. 26, $n^{\circ}$ 1, 2010, pp. 7-16. 35. Oumma.com, Nassurdine Haidari, « Nous ne marcherons plus! », 12 novembre 2011, http://oumma.com/Nousne-marcherons-plus, version du 12 novembre 2011, in Archives de l'Internet, Dépôt légal du numérique, BnF, également disponible en ligne, consulté le 8 octobre 2015. 36. Ibrahima Kone, « France : des jeunes perpétuent la Marche des beurs 25 ans après », Yabiladi. com, 2 juillet 2008, http://www.yabiladi.com/article-societe-3316.html, version du 11 juillet 2009, in Archives de l'Internet, Dépôt légal du numérique, BnF; Sylvia Zappi, « Rachid taxi ", de la Marche des Beurs à 2012, la même rage pour l'égalité, http://banlieue.blog. lemonde.fr/2013/01/18/rachid-taxi-de-la-marche-des-beurs-a-2012-la-meme-rage-pour-legalite/, consulté le 10 mai 2013, in Archives de l'Internet, Dépôt légal du numérique, BnF. 37. Agence Français de Presse (AFP), "Il y a vingt ans, la marche des Beurs ", 3 décembre 2003, http://www.bladi.net/il-y-a-vingt-ans-la-marche-des-beurs.html, version du 22 décembre 2009, in Archives de l'Internet, Dépôt légal du numérique, BnF. 38. Voir notamment le blog de la journaliste Sylvia Zappi, « Rachid Taxi, de la Marche des Beurs à 2012, la même rage pour l'égalité ", Au centre, la banlieue, 18 janvier 2013, http://banlieue.blog.lemonde.fr/2013/01/18/ rachid-taxi-de-la-marche-des-beurs-a-2012-la-meme-rage-pour-legalite/, version du 24 janvier 2013, in Archives de l'Internet, Dépôt légal du numérique, BnF. 39. Farid Saidani, "zoe anniversaire de la Marche pour l'égalité ", 2013, https://www.facebook.com/ 3oeAnniversaireDeLaMarchePourlegalite?fref=ts, Page aujourd'hui clôturée, in Archives de l'Internet, Dépôt légal du numérique, BnF. 40. ZEP, Zone d'éducation populaire, Nique la France, 8 août 2010, http://www.youtube.com/watch?v=KdA2j40U7v8\&feature=youtu be_gdata_player, in Dépôt légal du Web média, Ina ; Amine Bentounsi, " 30 ans après la marche des Beurs Amine Bentounsi ", 7 mai 2012, http://www.dailymotion.com/video/xqno4m_30-ans-apres-la-marche-des-beurs-amine-bentounsi_news, in Dépôt légal du Web média, Ina. 


\section{/// Les temps forts de la médiatisation de la Marche en ligne dans le corpus étudié de 1999 à 2013}

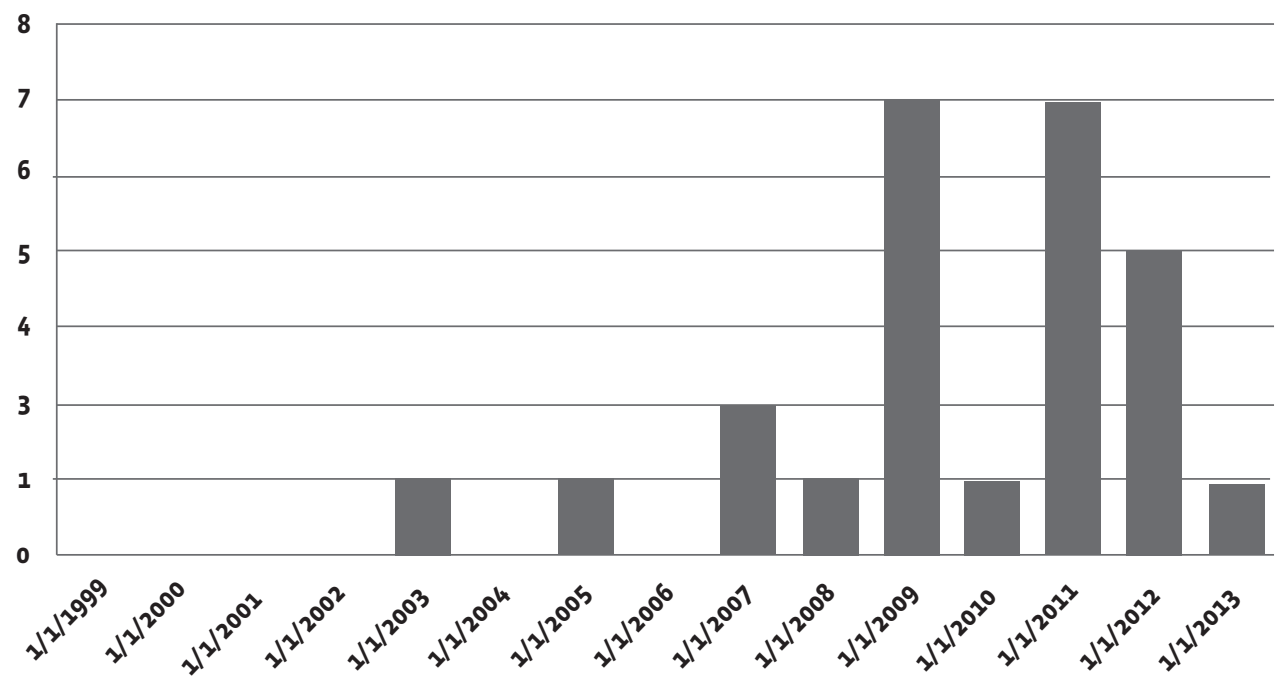

\section{Usages publics de la Marche en ligne : temporalités et mises en scène des témoins}

Au sein du corpus rassemblé, la Marche est perçue comme un événement délaissé par les médias traditionnels. L'un des premiers objectifs revendiqués est de sortir l'événement de l'oubli. Les temporalités de ces dispositifs reflètent l'impact des logiques commémoratives et confirment la place centrale accordée au témoin.

À l'échelle des années 2000, les temps commémoratifs sont l'occasion de faire connaitre l'existence de la Marche. Par exemple, pour les vingt ans de l'événement en 2003, le site Bladi.net publie une dépêche de l'AFP à ce sujet. Cependant, les temps forts de la mise en visibilité de la Marche de 1983 en ligne datent de 2009 et de 2011. Le premier correspond à la Marche de 2009, associant des anciens et des nouveaux marcheurs afin de porter de nouveau la question de l'égalité des droits dans le débat public. Le second temps est lié à l'appel de Nassurdine Haidari, élu socialiste marseillais, qui a fondé en 2011 le collectif Nous ne marcherons plus $^{41}$ !. À partir de 2012, la Marche de 1983 est «noyée » dans les multiples marches pour l'égalité liées au projet de loi sur le mariage pour les personnes de même sexe qui occupe alors l'actualité. La seule commémoration en ligne recensée à cette période prend la forme d'une page Facebook qui 
"Le MIB, un héritage des luttes de l'immigration ", in Archives de l'internet, Dépôt légal du numérique, BnF, 3 février 2002. D.R.

bénéficie des stratégies de référencements propres aux réseaux sociaux. Son auteur, Farid Saidani, directeur de centre social, attaché territorial dans le Val-d'Oise, a participé à la Marche de 1983 et à la Marche Convergence 84 l'année suivante ${ }^{42}$ avant d'intégrer l'association SOS Racisme. Lorsque nous l'avons questionné sur ses motivations en juin 2015, il déplorait l'absence de transmission de la mémoire de la Marche auprès des plus jeunes ${ }^{43}$. Au cœur de ces dispositifs, les passeurs de mémoire sont les marcheurs de 1983, acteurs-témoins de la Marche. La mise en visibilité des témoins est inégale et varie selon leur rôle dans la Marche de 1983 mais en fonction de leur propre pratique numérique. Ainsi, Toumi Djaidja et le père Christian Delorme, étant à l'initiative de la Marche, sont les plus visibles. Cela s'inscrit dans la continuité de la médiatisation de la Marche en 1983, notamment à la télévision. Pour Salika Amara et Saïd Bouamama, leur visibilité est étroitement liée à leur intense activité sur la Toile. Il convient d'ajouter les témoignages d'Abdelaziz Chambi, de Farida Belghoul, de Marie-Laure Mahé et de Rachid Amghar. Ces souvenirs sont le plus souvent diffusés sous la forme d'entretiens retranscrits ou, dans une moindre mesure, filmés. Les témoins racontent leur investissement dans la Marche et les espoirs qui sont nés de son succès. L'enthousiasme des récits est 
bien souvent associé à la dénonciation de la récupération politique par le Parti socialiste à travers la création, en 1984, de l'association SOS Racisme perçue comme un pion du PS. À ce désenchantement quasi immédiat lié au sentiment de confiscation du mouvement s'ajoute l'amer constat de la persistance de l'exclusion des enfants issus de l'immigration, notamment maghrébine. Dans ces récits de vie, la Marche constitue souvent l'entrée en résistance, le fondement d'une lutte et les premiers pas dans le militantisme. Ainsi, la majorité des témoins présents dans ces dispositifs ont poursuivi l'action militante, mais selon des trajectoires très diverses : Abdelaziz Chambi dirige l'Union des jeunes musulmans (UJM), Rachid Amghar s'implique dans la vie associative de son quartier du Blanc-Mesnil. Il s'agit d'un militantisme de terrain auprès des habitants des quartiers populaires à l'échelle micro-locale. Tout au plus, ces associations participent au forum social des quartiers populaires mis en place par le Mouvement de l'immigration et des banlieues. Au-delà du cadre associatif, l'engagement des marcheurs prend des formes variées au gré des personnalités et des opportunités à l'image du sociologue Saïd Bouamama, investi dans le mouvement postcolonial. Les marcheurs de 1983 apparaissent dans le corpus comme des figures héroïques discrètes, abîmées par une désillusion à la hauteur des espérances qu'avait soulevées leur implication dans la Marche pour l'égalité.

\section{Polyphonie mémorielle de la Marche}

Jusqu'en février 2013, le Web apparaît comme un espace de réhabilitation de la Marche dans la mémoire collective mais, au-delà de l'unanimité autour du caractère oublié de la Marche, la mémoire de l'événement est plurielle. Elle varie en fonction de son interprétation à la lumière des revendications portées par les différents acteurs. Cette plura- lité s'exprime au sujet de la dénomination, de l'héritage et de l'échec de la Marche. La confiscation est souvent associée au rejet de l'expression « Marche des Beurs ». Dans notre corpus, l'expression revient dans $43 \%$ des cas, alors que $57 \%$ privilégient la formule « Marche pour légalité (et contre le racisme) ». Pour les témoins, notamment Toumi Djaidja, l'appellation " Marche des Beurs » renvoie à une ethnicisation de «l'esprit de 1983 », qui noie les revendications dans la lutte antiraciste catalysée par SOS Racisme. L'expression renvoie également au traitement médiatique de l'événement centré sur la troisième génération de l'immigration maghrébine ${ }^{44}$. Ainsi, le Web militant privilégie l'expression " Marche pour l'égalité ", tout comme le média de minorité Oumma.com. Ces divergences de nomination révèlent une pluralité des interprétations de la Marche liée à des enjeux de filiation. Moment fon-
L'analyse des dispositifs mémoriels sur le Web au sujet de la Marche pour l'égalité et contre le racisme permet de constater que les acteurs de la mémoire en ligne convoquent le passé pour s'inscrire dans des modes d'action militants contemporains. Ces dispositifs relèvent davantage des usages du passé que de la volonté d'approfondir I'histoire de la Marche et sa contextualisation. dateur, la Marche est aussi une source de légitimité. Elle est convoquée par les différents acteurs du mouvement social qui souhaitent s'inscrire dans la continuité de ce moment clé du militantisme contemporain et s'inscrit donc dans des filiations multiples.

La première approche est celle de l'antiracisme universaliste incarné par SOS Racisme, dont le site fait de 1983 la première date de l'historique de l'association : «1983 : Marche des Beurs, crime raciste Bordeaux-Vintimille ${ }^{45}$ ». Ici lévénement est à la fois central, à l'origine de l'association, sans être pour autant l'objet d'un développement important, la présentation restant assez sommaire. À l'inverse, pour les militants du MIB, la Marche est insérée dans une temporalité plus longue : depuis le 17 octobre 1961 jusqu'au mouvement de soutien aux sans-papiers. 
Dans cette deuxième approche, la Marche est perçue comme un moment de révolte contre les violences policières ${ }^{46}$ tout en dénonçant les mouvements antiracistes décrits comme paternalistes ${ }^{47}$. Il s'agit donc aussi, plus largement, de réhabiliter les luttes de l'immigration dans la mémoire collective. Pour SOS Racisme, la Marche est un événement qui trouve son aboutissement dans la création de l'association, tandis que, pour le MIB, comme pour les associations locales, elle apparait davantage comme un échec. Dans le même temps, la situation des grands ensembles, le traitement politique de l'immigration et les discriminations, trente ans après, renforcent le sentiment d'échec de la Marche. Ainsi, Abdelaziz Chambi dénonce une accentuation de l'ethnicisation des rapports sociaux depuis les années 1980 ${ }^{48}$. Enfin, la Marche est aussi insérée dans une lecture postcoloniale ${ }^{49}$ diffusée notamment par le MIR dès 2005. Dans cette perspective, il s'agit de dénoncer la continuité des pratiques coloniales de l'État français à l'égard de populations perçues comme «non blanches ». La vidéo du clip Nique la France, mise en ligne sur Youtube, dénonce l'incapacité de la France à sortir du regard colonial concernant les populations issues de l'immigration. Sur un texte du marcheur et sociologue Saïd Bouamama, proche du MIR, le chanteur Saïdou dresse un tableau désenchanté du quotidien de l'exclusion dans lequel l'échec de la manifestation de 1983 symbolise l'absence d'écoute à l'égard des populations ghettoïsées. L'héritage de la Marche est ici davantage celui d'un échec sur la durée. Elle est à la fois le symbole d'une mobilisation collective et celui des difficultés rencontrées dans le dialogue avec les gouvernements successifs.

\section{Conclusion}

L'analyse des dispositifs mémoriels sur le Web au sujet de la Marche pour l'égalité et contre le racisme permet de constater que les acteurs de la mémoire en ligne convoquent le passé pour s'inscrire dans des modes d'action militants contemporains. Ces dispositifs relèvent davantage des usages du passé que de la volonté d'approfondir l'histoire de la Marche et sa contextualisation. Les acteurs mémoriels trouvent ainsi dans le Web un nouvel espace de mise en visibilité d'une mémoire considérée comme oubliée, facilitant ainsi la transmission et les processus de réappropriation. Finalement, malgré de réelles oppositions entre les héritiers de la Marche, le Web apparaît davantage comme un espace de coexistence dans lequel chaque acteur mémoriel tente d'exister à différentes échelles. La Toile montre ainsi une mémoire territorialisée de la Marche, où les militants locaux cohabitent avec les associations et les institutions d'envergure nationale. Aux côtés des témoignages narrant l'arrivée triomphale de la Marche à Paris en décembre 1983, les archives du Web donnent aussi accès à la mémoire micro-territorialisée de la Marche qui s'inscrit dans la pratique numérique de la vie associative des quartiers urbains qui furent des lieux d'impulsion de l'événement, qu'il s'agisse de Marseille ou de Lyon. La pluralité des mémoires et la récupération politique de la Marche témoignent plus largement de la difficulté d'édifier des événements référents, fédérateurs et unanimes, à partir de mémoires migrantes.

46. Voir également Anonyme, "Ratonnages, chronologies », http://infokiosques.net/imprimersans2.php?id_article=677, version du 24 novembre 2011, in Archives de l'internet, Dépôt légal du numérique, BnF, également disponible en ligne, consulté le 2 septembre 2015 ; et Amine Bentounsi, op. cit. 47. Sur le militantisme immigré et anti-racisme, voir notamment : James Robert House, "Antiracism and antiracist discourse in France from 1900 to the present day", phd, University of Leeds, 1997. 48. Patrice Berger, " 25 ans de marche, et toujours pas d'égalité ", 10 avril 20og, http://www.radiopluriel.fr/spip/25-ans-de-marche-et-toujours-pasd.html, version du 23 mai 2011, in Archives de l'Internet, Dépôt légal du numérique, BNF, également disponible en ligne, consulté le 2 septembre 2015. 49. Fiona Barclay, France's Colonial Legacies Memory, Identity and Narrative, Cardiff, University of Wales press, 2013. 